\title{
Enhancement of open-cracks detection using a principal component analysis/wavelet technique in photothermal nondestructive testing
}

\author{
by S. Hermosilla-Lara ${ }^{1,2,3}$, P.Y. Joubert ${ }^{2}$, D. Placko², \\ F. Lepoutre ${ }^{3}$, M. Piriou
}

(1) Technical Center of Framatome, ZIP Sud 71380 Saint-Marcel, France,

(2) Laboratoire Electronique Signaux et Robotique (LESiR), Ecole Normale Supérieure de Cachan, 61 av. du Président Wilson, 94235 Cachan, France,

(3) Dept of Structure and Damage Mechanics, ONERA, 29 avenue de la Division Leclerc BP72, 92322 Châtillon cedex, France - e-mail: lepoutre@onera.fr

\begin{abstract}
In this paper, the images provided by a flying-spot camera dedicated to open-crack detection are considered. In this contribution, the authors focus on the enhancement of open-crack detection performances in the case of severe surface conditions. After a short description of the principle of the device, the tested structure and the obtained photothermal images are presented. The images are then processed thanks to a modified principal component analysis which allows to separate the thermal and optical effects from the raw images. The detection is then carried out and the performances are characterized thanks to Receiver Operating Characteristic curves.
\end{abstract}

\section{Introduction}

Among the existing non-destructive testing techniques, the active thermography appears to be a good alternative to penetrant testing for open-crack detection in metallic structures, because it is a contactless and automatizable technique. A photothermal camera prototype (flying-spot camera) was realized and presented in [1] and is implemented here for the testing of an industrial mockup showing open fatigue cracks with severe surface conditions. After a short description of the basic principle of the implemented flying-spot camera, the mockup and the obtained photothermal images are presented. Then an image processing method, based on modified principal component analysis is implemented in order to facilitate the open crack detection. The detection performances are finally evaluated in the case of different surface conditions, using Receiver Operating Curves.

\section{The flying-spot camera}

\subsection{Principle}

The flying-spot camera used in this study was presented in [1]. It consists in a simple and portable device, composed of a laser used as a thermal excitation source and an array of infrared detectors as thermal sensors. The images are obtained in an active thermography scheme [1] thanks to a simultaneous scanning of the inspected structure surface by the excitation and detection beams at a constant speed and with different offsets (Figure 1).

Two main physical phenomena take place in this type of active thermography: the thermal effect which is a thermal barrier effect produced by the presence of a crack, and the optical effects which mainly consist of a variation of the absorptivity or diffusivity factor of the inspected surface. The signals provided by the camera are resulting from a combination of all these effects, the proportions of the combination depending on the nature of the defects and of the inspected surface. The combination is assumed to be 
linear and this assumption was experimentally verified. In the relatively rare case of opencracks with aperture smaller than $20 \mu \mathrm{m}$ on a surface with a good absorptivity, the thermal barrier is the dominating effect, and the defect signal leads to an easily interpretable signature, called "thermal signature". In the case of surface defects such as corrosion areas, rough surface etc, the dominating effects are the optical ones, which produce "optical signatures". In the rather common case of open-cracks on metallic structure showing rough and rusty surface conditions, optical and thermal effects are competitive and lead to hardly interpretable signatures which make the technique hardly efficient if applied without any processing. In what follows, the images obtained by the inspection of a mockup containing open fatigue cracks and showing different surface conditions are considered.

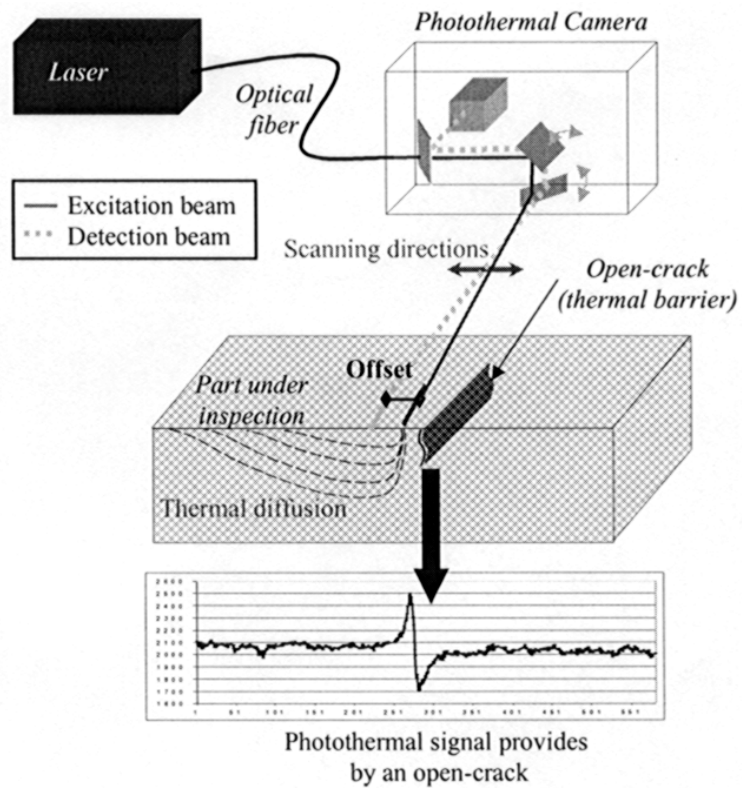

Fig. 1. Basic principle of the photothermal camera for open-crack detection

\subsection{Inspected mockup, photothermal images and first improvement}

The inspected structure is a laboratory-made test structure featuring various surface conditions (glossy, oxidized, ground...), and different defects such as fatigue open-crack, grooves and holes, as seen on the photography of the structure shown in Figure 2a. The raw photothermal image is given in Figure $2 \mathrm{~b}$.

The first processing method, called normalization was proposed in [2]. The normalization is based on the morphological differences between the thermal and optical signatures, according to the scanning direction of the structure under test for a given offset. Indeed, by subtracting the raw image obtained by a forward scanning from the raw image obtained by a backward scanning, a resulting image is obtained showing highlighted thermal signatures (Figure $2 \mathrm{~d}$ ), since the subtraction is mainly constructive in the case of thermal signatures and destructive in the case of optical signatures. However, this technique allows to enhance only the thermal effect at each offset [1].

In this paper, in order to improve the crack detection, the authors generalize this technique in a multi-image scheme to separate the physical effects. The proposed method is based on a principal component analysis (PCA), applied to the whole set of photothermal images of different offsets. 

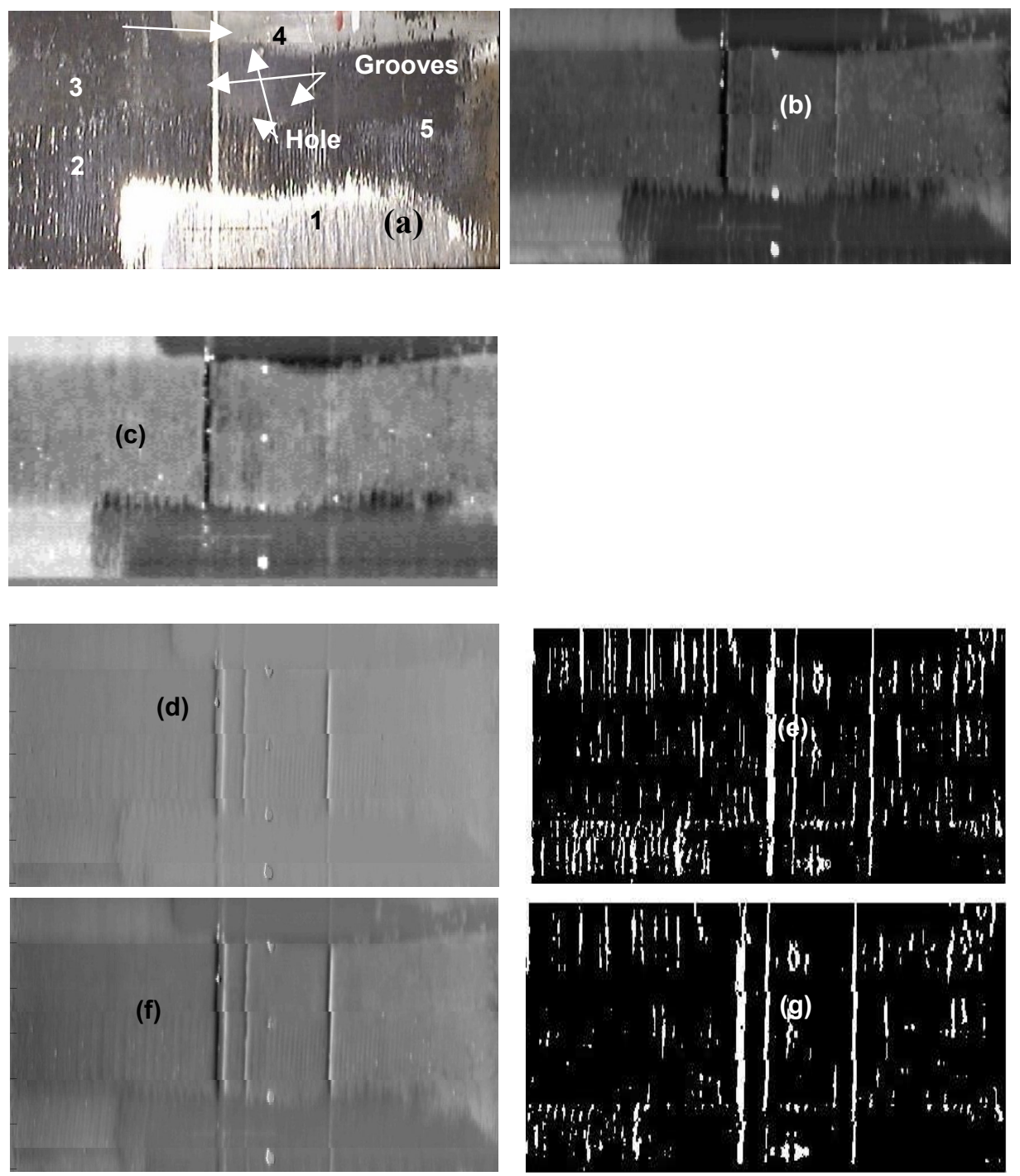

Fig. 2. Laboratory-made structure with fatigue open-crack

(a) photography of the structure, zone $n^{\circ} 1$ : ground and glossy, zone $n^{\circ} 2$ : ground and oxidized, zone $n^{\circ} 3$ : polished and oxidized, zones $n^{\circ} 4$ and $n^{\circ} 5$ : polished and glossy;

(b) raw photothermal image;

(c) retrieving of the optical effect;

(d) normalized image;

(e) thresholding of normalized image (white pixel: crack detection, black pixel: no defect);

(f) retrieving of the thermal image;

(g) threshold of the thermal image (white pixel: crack detection, black pixel: no defect). 


\section{Physical effects separation}

\subsection{Principle of principal component analysis}

Let us define $M$ as a matrix of raw data measurement $m_{i}$ provided by the camera. The signals of $M$ result from the combination of the physical source $s_{i}$, (thermal effect, optical effects, noise...) through the unknown transfer matrix $T$ of the camera. If $r$ sources $s_{i}$ constituting the source matrix $\Sigma$ are considered and $p>r$ measures signals $m_{i}$ are available, the matrix $M$ can be written as:

$$
M=\left[\begin{array}{c}
m_{1} \\
m_{2} \\
\cdots \\
m_{p}
\end{array}\right]=\underset{p \times r}{T} \cdot\left[\begin{array}{c}
s_{1} \\
s_{2} \\
\cdots \\
s_{r}
\end{array}\right]=\underset{p \times r}{T} \cdot \Sigma
$$

The PCA separates the different sources constituting the signals of the matrix $M$. This separation is realized by the projection of $M$ on the subspace formed by the eigenvectors of the covariance matrix $M M^{t}[3]$, as expressed by:

$$
\left(Q^{A C P}\right)^{t} M=\left[\begin{array}{cccc}
\left\|t_{j_{1}}\right\| & 0 & \cdots & 0 \\
0 & \left\|t_{j_{2}}\right\| & \cdots & 0 \\
\vdots & \vdots & \ddots & \vdots \\
0 & 0 & \cdots & \left\|t_{j_{r}}\right\|
\end{array}\right] S=\left[\begin{array}{c} 
\pm\left\|t_{j_{1}}\right\| s_{j_{1}}{ }^{\prime} \\
\pm\left\|t_{j_{2}}\right\| s_{j_{2}}{ }^{\prime} \\
\vdots \\
\pm\left\|t_{j_{r}}\right\| s_{j_{r}}{ }^{\prime}
\end{array}\right]=R^{A C P}
$$

where $Q^{A C P}$ is the eigenvectors matrix of $M M^{t}$ classified by descending order, $\left\|t_{j_{i}}\right\|$ is the norm of the $j_{i}{ }^{\text {th }}$ column of $T$. The lines of the result matrix $R^{A C P}$, called components of $R^{A C P}$, are constituted of the separated sources obtained within the multiplicative factor $\pm\left\|t_{j i}\right\|$. However, if the sources are separated they are not identified, that is to say one does not a priori know on which component of $R^{A C P}$ the different physical effects will appear. But by judiciously choosing the covariance matrix $M M^{t}$ it is possible to force a particular physical effect to appear on the first component of $R^{A C P}$ as describe in the following section.

\subsection{Retrieving of the optical effects}

Choosing a specific raw data measurement matrix $M_{o p}$ essentially relative to optical effects, the first component of the result matrix $R_{o p}^{A C P}$ will inevitably correspond to the optical effects. Therefore, the first vector $V_{o p}$ of the eigenvectors matrix $Q_{o p}^{A C P}$ is the best projection operator for retrieving the optical effects. The optical effects of the whole photothermal image set is then obtained by the projection of the whole image set along $V_{\text {op. }}$.

Here, the matrix $M_{o p}$ is built by the inspection of a reference surface containing a black line drawn with a pencil, i.e. a high absorptivity effect without thermal effect.

The result of the projection of the image set onto $V_{o p}$ is shown in Figure 2c. It can be seen that the optical effects (due to glossy and/or ground areas, holes, grooves...) are clearly visible independently from the thermal effect.

\subsection{Retrieving of the thermal effect}

The components provided by the PCA are orthogonal to each other [3]. The optical and the thermal effects are assumed to be independent - this assumption was experimentally verified - therefore they will appear on separate components of the PCA. Since the optical effects appear on the first component, the thermal effect will be retrieved 
on one of the secondary components [4]. In order to identify the component containing the thermal effect, the knowledge of the thermal signature morphology - as given by [2,5] was used. In this study, the thermal effect appears on the $2^{\text {nd }}$ component, since its energy contribution is the second one of the total energy of the considered signal.

\section{Application to Open-crack Detection}

The detection consists in obtaining a binary image representative of the presence of open-cracks. The detection is implemented using a 1D Continuous Wavelet Transform (CWT) applied on the obtained thermal effect image $I_{\text {th }}$ shown in Figure $2 f$. For each line $I_{t h}^{\prime}(x)$ of $I_{t h}$, the obtained wavelets coefficients $c_{a}^{\text {th }}$ are expressed by [6]:

$$
c_{a}^{\text {th }}(b)=\int_{-\infty}^{+\infty} I_{t h}^{l}(x) \cdot \psi_{a, b}^{*}(x) \cdot d x
$$

where $x$ denotes the spatial position, a denotes the dilatation scale, $b$ the spatial shift. The wavelet set $\psi_{a, b}(x)$ is built from the mother wavelet $\psi(x)$ by means of time shift and dilatation, as expressed by:

$$
\psi_{a, b}(x)=\frac{1}{\sqrt{a}} \psi\left(\frac{x-b}{a}\right)
$$

As the thermal effect exhibits a bipolar signature, the mother wavelet $\psi(x)$ is chosen to be a first derivative of a gaussian, because of its shape similarity.

The crack-detection image is a binary image resulting from the following decision scheme applied on each line:

$$
d_{a}^{\text {th }}(b)=\left\{\begin{array}{l}
1 \text { if } c_{a}^{\text {th }}(b) \geq \lambda \\
0 \text { otherwise }
\end{array}\right.
$$

where $\lambda$ is a threshold value.

The detection performances are evaluated in different areas of the mockup, showing various surfaces conditions, such as glossy or oxidized, ground or polished as seen in Figure 2a. The best detection performances are obtained for surfaces showing oxidized and polished conditions, because the surface noise and the optical reflections are minimum, and the absorption coefficient is great. The worst case of detection is obtained for glossy surfaces, because of the high optical reflection coefficient.

In each zone, the detection performances are characterized using Receiver Operating Characteristic (ROC) curves [7]. The ROC curves exhibit the Good Detection Rate (GDR), defined as the ratio of the detected cracks to the actual number of crack, versus the False Alarm Rate (FAR) defined as the ratio of false cracks actually detected to the number of false cracks possibly detected (Figure 3 ). Best detection performances are obtained when the distance of the curve to the point $(0,1)$ is minimum. In each zone, both values of $\lambda$ and $a$ were chosen in order to minimize this distance.

As seen on the ROC curves shown in Figure 3, the detection performances reach $97 \%$ GDR for $4 \%$ FAR in the case of oxidized areas (best surface conditions for crack detection) when the thermal image is considered. In the case of the normalized image, the performances are $91 \%$ GDR for $5 \%$ FAR in the same areas.

In the case of glossy areas (worst surface conditions for crack detection) the detection performances reach 95\% GDR for 10\% FAR when applied on the thermal image, and decrease to $85 \%$ GDR for $15 \%$ FAR when applied to the normalized image.

As illustrated in Figure 3, the detection performances are increased when the thermal image is used instead of the normalized one, whatever the surface conditions. 


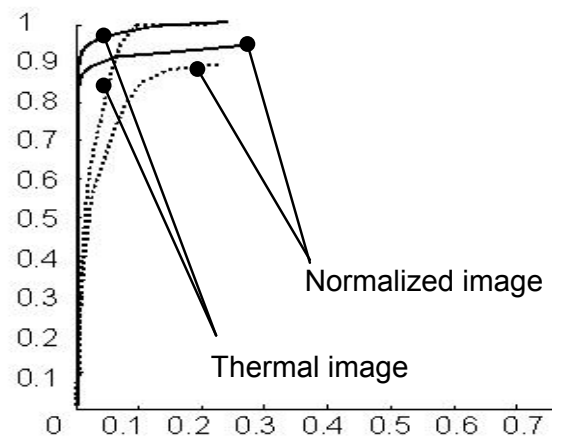

a.

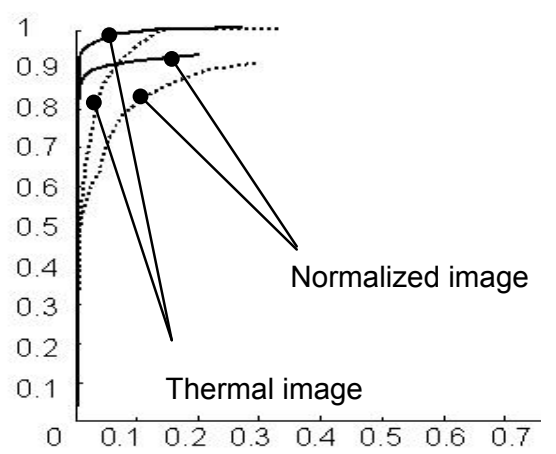

b.

Fig. 3. ROC curves for open crack detection (Dashed curves: glossy areas, continuous curves: oxidized areas); a: ground zone, b: polished zone.

\section{Conclusion}

In this paper, photothermal images were considered for the detection of open cracks in a metallic structure. Since the thermal effect is essentially relative to open-cracks, two methods of thermal effect enhancement were implemented in order to improve the crack detection: the image normalization, and the PCA. The detection performances obtained when using both enhancement methods were compared thanks to ROC curves. The comparison was carried out in different areas of the structure featuring various surface conditions. In each considered cases, the method based on PCA allows to improve the detection performances. These results show the benefit of combining multiple photothermal images obtained at various offsets. In further work, the implemented PCA method could be extended to a physical effect identification using a priori known signatures of the different considered physical effects.

\section{REFERENCES}

[1] C.Gruss, D. Balageas, "Theoretical and experimental applications of the flying spot camera”, Quantitative Infrared Thermography, D. Balageas et al. (Eds.), Ed. Européennes Thermique et Industrie, 1992, p. 19-24.

[2] Legrandjacques L., Krapez J.C., Lepoutre F. and Balageas D. "Nothing but the Cracks: A New Kind of Photothermal Camera", 7th European Conference on NonDestructive Testing, Copenhagen, Denmark, pp. 2651-2656, 26-29 May 1998.

[3] Saporta G., Probabilités, Analyse de données et statistique, Technip, 1990 [in french].

[4] J.C. Krapez, F. Lepoutre, R.Huttner, C. Gruss, L. Legrandjacques, M. Piriou, J. Gros, D. Gente, S. Hermosilla-Lara, P.-Y. Joubert, D. Placko. "La caméra photothermique, partie II - Applications industrielles, perspectives d'amélioration par un nouveau traitement d'image". Revue I2M (Instrumentation, Mesure et Métrologie), Special issue on Non Destructive Evaluation, Hermès Publications, Paris, vol. 1, n¹-2, 2001, pp.41-67. [in french]

[5] Krapez J.-C., "Spatial resolution of the flying spot camera". Int. J. Thermal Sci., vol. 38, 1999, p. 769-779.

[6] Cohen, J. Kovacevic, "Wavelets the Mathematical Background". Proceedings of the IEEE, vol. 84 n4, pp. 514-522. April 1996.

[7] Ergan J.P., "Signal detection theory an ROC analysis", Series in cognition and perception, New-York: Academic Press, 1975. 